\title{
The role of lipids in the biogenesis of integral membrane proteins
}

\author{
Roger Schneiter • Alexandre Toulmay
}

Received: 29 August 2006 /Revised: 3 October 2006 / Accepted: 4 October 2006 / Published online: 17 November 2006

(C) Springer-Verlag 2006

\begin{abstract}
Most integral membrane proteins are cotranslationally inserted into the lipid bilayer. In prokaryotes, membrane insertion of the nascent chain takes place at the plasma membrane, whereas in eukaryotes insertion takes place into the endoplasmatic reticulum. In both kingdoms of life, however, the same membrane that acquaints the newly born membrane protein also synthesizes the bilayer lipids and thus ensures the balanced growth of the membrane as a whole. Recent evidence indicates that the lipid composition of the host membrane can determine the fate of the newborn membrane protein, as it can affect (1) the efficiency of translocation, (2) the topology of the resulting membrane protein, (3) its stability, (4) its assembly into oligomeric complexes, (5) its transport and sorting along the secretory pathway, and (6) its enzymatic activity. The lipid composition of the membrane thus can affect the biogenesis and function of integral membrane proteins at multiple steps along its biogenetic pathway. While understanding this interdependence between bilayer lipids and protein biogenesis is interesting in its own right, careful consideration of a potential host's membrane lipid composition may also allow optimization of the yield and activity of membrane proteins that are expressed in a heterologous organism. Here, we review and discuss some examples that illustrate the interdependence between bilayer lipids and the biogenesis of integral membrane proteins.
\end{abstract}

Keywords Protein translocation and transport . Membrane microdomains (rafts) · Secretory pathway .

R. Schneiter $(\bowtie) \cdot$ A. Toulmay

Department of Medicine, Division of Biochemistry, University of Fribourg,

Chemin du Musée 5,

CH-1700 Fribourg, Switzerland

e-mail: roger.schneiter@unifr.ch
Plasma membrane $\cdot$ Lipids $\cdot$ Sterols $\cdot$ Sphingolipids . Fatty acids $\cdot$ Saccharomyces cerevisiae

\section{Introduction}

Cellular membranes form the essential permeability barrier that separates the interior of the cell and its organelles from their surroundings. The exchange of nutrients and information across these barriers is enabled by the action of integral membrane proteins. Integral membrane proteins account for approximately $20-30 \%$ of the proteome of prokaryotic or eukaryotic organisms (Krogh et al. 2001). Their biogenesis and enzymatic function is tightly linked to that of the membrane itself and to the lipid composition of the respective membrane. Here, we review evidence that illustrates how lipids can affect the biogenesis and function of integral membrane proteins. The given examples highlight the dynamic interplay between proteins and lipids and are important to understand membrane biogenesis per se, that is the coordinated growth of both protein and lipid constituents of a cellular membrane.

Most integral membrane proteins are cotranslationally inserted into the lipid bilayer through a protein-aqueous channel that is located either in the endoplasmatic reticulum (ER) membrane of eukaryotic cells or the plasma membrane of eubacteria and archaea. A conserved heterotrimeric membrane complex, the Sec61 complex in eukaryotes, and the SecY complex in bacteria form this translocon. The translocon works in concert with bound ribosomes to recognize emerging transmembrane helices of 15-20 amino acid residues in length based on their hydrophobicity and releases them through a lateral opening into the lipid bilayer (Van den Berg et al. 2004; Hessa et al. 2005). Within the bilayer, individual helices of a polytopic protein then assemble into helical bundles and the conformation of the protein further matures to acquire its free energy minimum 
(Bowie 2005). The energy for translocation is derived from GTP hydrolysis and translocation requires an electrochemical gradient across the membrane. The topology of the newborn protein generally follows the positive-inside rule to position lysine and arginine residues flanking the transmembrane domain into the cytosol (Osborne et al. 2005). Once properly inserted into its lipid environment and matured in the ER, the transmembrane protein may be packed into vesicular carriers that are then transported and sorted to one of the membranes of the secretory pathway.

In both prokaryotic and eukaryotic cells, the membrane that synthesizes integral membrane proteins also harbors the lipid biosynthetic machinery. The colocalization of these two process is likely not incidental but a means to ensure the balanced growth of both lipid and protein constituents of a membrane. Striking examples of this coordination are observed upon overexpression of integral membrane proteins, which results in the induction of surplus membranes to accommodate the overexpressed protein (Wright et al. 1988). Lipids, however, are also more directly implicated in the membrane insertion and translocation process, as many of the components of the assembly machinery are known to be lipid-dependent. Anionic phospholipids are required for the proper topological orientation of the newly formed transmembrane protein (van Klompenburg et al. 1997; van Dalen and de Kruijff 2004).

The lipid composition of a cellular membrane profoundly affects the physicochemical properties of the bilayer and, thereby, the activity and function of integral membrane proteins. The hallmark of the lipid composition of cellular membranes is their complexity, as these membranes are typically composed of lipids with many different types of headgroups and acyl chains (Dowhan 1997; Epand 1998). This lipid diversity is likely to be important to ensure the dynamic functioning of membranes under changing environmental conditions, and may also account for the differences in function that the various subcellular membranes have to fulfill. In addition to bulk bilayer lipids, specific lipids have been seen in many crystal structures of integral membrane proteins and are often crucial for protein function. For example, cytochrome $\mathrm{C}$ oxidase is inactivated by the removal of cardiolipin and the bacterial potassium channel KcsA requires anionic phospholipids for ion transport (Lee 2004; van Dalen and de Kruijff 2004). Thus, lipids can act as cofactors for some membrane proteins and can stabilize their structures (Jensen and Mouritsen 2004; Palsdottir and Hunte 2004).

Given that protein and lipid biosynthetic machineries colocalize within the same cellular compartment and given their mutual interdependence, one wonders of how they coordinate their activities. This coordination is still poorly understood on a global scale. However, selected examples serve to illustrate the interdependence between the lipid composition of a membrane and its capacity to synthesize and mature integral membrane proteins. In this minireview, we will mainly focus on two aspects of this protein-lipid interdependence. The first illustrates the requirement for specific membrane lipids for the correct topological insertion of the nascent chain into the bilayer, as exemplified by the lactose permease of Escherichia coli, LacY. The second example illustrates a role of lipids with unusually long acyl chains in the transport of the abundant proton pumping ATPase to the cell surface of yeast.

\section{Phosphatidylethanolamine is required for a functional topology of the lactose permease, LacY, in $E$. coli}

One of the most striking and, also, best-characterized interplays between membrane phospholipid composition and the structure and function of an integral membrane protein is that of the lactose permease LacY from $E$. coli (Dowhan et al. 2004). LacY is a particularly well-studied representative of the major facilitator superfamily (MFS) transporters and a member of the oligosaccharide/proton symporter subfamily of the MFS transporters. Like many MFS members, LacY couples the free energy released from the downhill translocation of protons in response to an electrochemical proton gradient to drive the energetically uphill stoichiometric accumulation of galactosides against a concentration gradient. The molecule is composed of $\mathrm{N}$ and C-terminal domains, each with six transmembrane helices, symmetrically positioned within the permease. Common to many sugar permeases, including LacY, is a very hydrophilic transmembrane domain 7 that is not predicted by membrane topology algorithms but has been verified biochemically (Abramson et al. 2003).

Phosphatidylethanolamine (PE) is the most abundant phospholipid of the inner membrane of E. coli, where it constitutes $75-80 \%$ of total phospholipids. It is a nonbilayer-forming lipid because it has a small polar headgroup relative to the diameter occupied by the two acyl chains. This small headgroup gives the lipid the shape of a cone when rotated along its long axes. Such cone-shaped lipids form inverted hexagonal phases characterized by high local curvature rather than bilayers. When incorporated into a bilayer together with other lipids, PE evokes lateral pressure, which is thought to stabilize membrane proteins in their native conformation (Epand 1998).

Earlier studies in the mid-eighties in which LacY was reconstituted in proteoliposomes of various lipid compositions revealed that $\mathrm{PE}$ is required for $\mathrm{LacY}$ activity in vitro (Chen and Wilson 1984; Seto-Young et al. 1985). Subsequent in vivo studies revealed that LacY assembled in $E$. coli mutant lacking PE cannot accumulate substrate against a 
concentration gradient, but it can still facilitate substrate transport/diffusion (Bogdanov and Dowhan 1995). Loss of full transporter function of $\mathrm{LacY}$ in cells lacking PE correlates with a structural alteration in the periplasmic domain $\mathrm{P} 7$ of LacY, as indicated by a loss of recognition by a conformation-sensitive monoclonal antibody (Fig. 1). PE is not required for membrane insertion of LacY, but it is required to facilitate the proper folding and membrane topology of LacY late in the maturation process. A systematic comparison of the transmembrane topology of $\mathrm{LacY}$ in cells containing or lacking PE revealed a topological inversion of the N-terminal half of LacY when the protein is assembled in the absence of PE. Remarkably, however, active transport by LacY and reversion of the inverted topology can be restored by addition of PE even after LacY has been synthesized and inserted into the membrane. These results indicate that LacY can adopt two different topologies, depending on whether the membrane contains PE or not, and that the two topologies are interchangeable postinsertionally in response to phospholipid composition. The results thus implicate phospholipids as specific participants in determining membrane protein organization and have been taken to suggest that the regulation of membrane protein function can occur by topology "switching" in response to changes in the phospholipid environment (Bogdanov et al. 2002; Wang et al. 2002).

The in vivo and in vitro studies on LacY topology are consistent with a role of PE to act as a molecular chaperone in the folding of this polytopic membrane protein. The proposed chaperone function of PE in establishing the correct topological orientation of transmembrane domain 7 in LacY appears to be independent of the ability of this lipid to form inverted hexagonal phases as PE with two saturated acyl chains could correct the folding defect of LacY in vitro, whereas $\mathrm{PE}$ species that do not support bilayer formation failed to correct the folding defect (Bogdanov et al. 1999). Thus, the ability of PE as an overall neutral, but zwitterionic, lipid to form hydrogen bonds with amino acid residues appears to be critical for its chaperone activity. Remarkably, PE can be replaced by a foreign lipid, monoglucosyldiacylglycerol, to restore the uphill transport and wild-type topology of LacY. Thus, the two most abundant lipids in Gram-negative and Gram-positive bacteria have an interchangeable role in defining the transmembrane domain orientation of $\mathrm{LacY}$ and possibly other integral membrane proteins (Xie et al. 2006). LacY is not the only integral membrane protein whose topology is sensitive to PE levels, as similar observations where reported for the high-affinity phenylalanine permease and the gamma-aminobutyric acid permease, both belonging to the amino acid/polyamine/organocation superfamily of secondary transporters (Zhang et al. 2003, 2005).

The transmembrane topology of most eukaryotic polytopic membrane proteins is established cotranslationally at the ER membrane and is maintained during subsequent steps of folding and transport. The biogenesis of these proteins involves a series of coordinated translocation and membrane integration events that is directed by topogenic determinants within the nascent chains and that ultimately leads to a uniform topology for any given polypeptide. In recent years, however, it has become evident that certain cellular polytopic proteins exhibit variations in biogenesis such that two or more distinct topological orientations are generated (Levy 1996; Hegde and Lingappa 1999). Remarkably, a recent global analysis of the topology of the inner-membrane proteome of $E$. coli revealed five candidate proteins that exhibited two alternative topologies (Rapp et al. 2006). The X-ray structure of one of these, EmrE, a member of the small multidrug resistance family of efflux transporters, provides conclusive evidence for its dual topology (Pornillos and Chang 2006). It will be interesting to learn whether the relative topology of these membrane proteins is modulated by the lipid composition of the bilayer.

There are a number of other examples of integral membrane proteins that are expressed in alternate topological forms with the diversity apparently generated at the time of translocation at the ER membrane. These include the multidrug resistance P-glycoprotein (Zhang et al. 1993), the transporter ductin (Dunlop et al. 1995), the aquaporin-1 water channel protein ( $\mathrm{Lu}$ et al. 2000), and the hepatitis B
Fig. 1 Topology of the E. coli lactose transporter LacY in cells containing or lacking PE. The $\mathrm{N}$-terminal seven transmembrane domains of LacY switch their membrane orientation in the absence of PE (adapted from Dowhan et al. 2004)

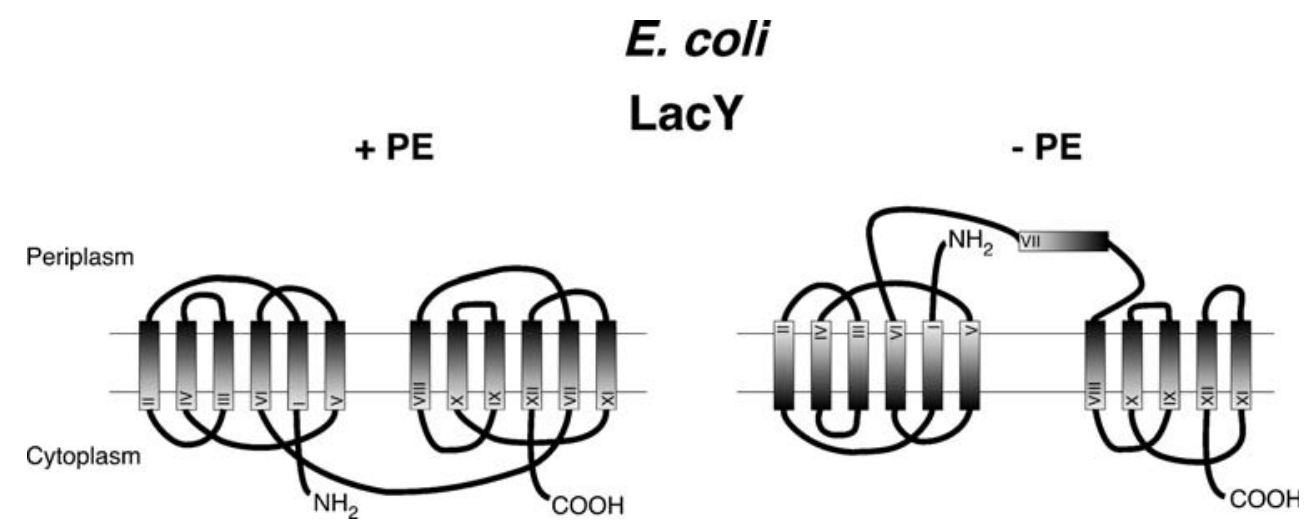


virus large envelope protein (Lambert and Prange 2001). On the other hand, OEP7, an envelope protein of chloroplasts, orients with native topology in liposomes that reflect the in vivo lipid composition, but with an opposite topology in liposomes of nonnative composition (Schleiff et al. 2001). Given the fact that proteins can assume alternative topologies in wild-type cells, and that lipid mutants display alternative topologies of some integral membrane proteins, it seems conceivable that the lipid composition of the bilayer or transport of the protein to a different subcellular membrane may serve to modulate protein topology and, thereby, protein function.

\section{Phosphatidylethanolamine is required for the transport of the arginine permease, Can1, to the cell surface of yeast}

In yeast, $\mathrm{PE}$ constitutes approximately $20 \%$ of the total phospholipids and a minimal level of $\mathrm{PE}$ is required for viability because depletion of PE below $4 \%$ affects the growth of the organism on nonfermentable carbon sources or at elevated temperatures (Birner et al. 2001; Storey et al. 2001). Depletion of PE in yeast affects the uptake of nutrients such as arginine, mediated by Can 1 ; uracil, mediated by Fur4; proline, mediated by Put4; general amino acid uptake, mediated by Gap1; and uptake of maltose, mediated by Mal6. Uptake of glucose by the hexose transporter, Hxt1, on the other hand, is not affected (Robl et al. 2001; Opekarova et al. 2002). Interestingly, however, in PE-depleted cells, Can1 accumulates in the Golgi apparatus instead of being transported to the plasma membrane. Arrest of Can1 in the Golgi appears not to be due to a folding defect of the protein, as Can1 function can be restored in vitro by reconstitution of the protein into liposomes containing PE (Opekarova et al. 2005). In wild-type cells, Can1 is associated with detergentresistant membranes, or lipid "rafts," and is localized in discrete domains at the cell surface that are distinct from those occupied by the abundant proton pumping $\mathrm{H}^{+}$-ATPase (Malinska et al. 2003). Interestingly, depletion of PE impairs raft association of Can1, but not that of the ATPase, indicating that the lipid environment of Can1 is distinct from that of the ATPase and that the two proteins react differently on changes in that environment (Opekarova et al. 2005).

\section{Very-long-chain fatty acid-containing lipids are required for stable surface transport of the proton pumping $\mathrm{H}^{+}$-ATPase in yeast}

The proton pumping $\mathrm{H}^{+}$-ATPase, Pma1, is an abundant and long-lived polytopic membrane protein of the yeast plasma membrane. The proton pumping activity of Pmal is essential for regulating intracellular $\mathrm{pH}$ and for uptake of nutrients by plasma membrane symporters. The protein belongs to the family of cation-transporting ATPases, which includes the $\mathrm{Na}^{+} / \mathrm{K}^{+}$-ATPase and $\mathrm{Ca}^{2+}$-ATPase of the mammalian plasma membrane (Kühlbrand 2004). Pmal accounts for more than $25 \%$ of all the proteins of the plasma membrane and thus constitutes a major cargo of the secretory pathway. Pmal serves as an excellent model to study the biogenesis of the plasma membrane, that is the coordinated synthesis, assembly, and transport of both the protein and lipid constituents of this membrane.

Pmal is biosynthetically inserted into the membrane of the ER, from where it is transported by vesicular carriers to its final destination (Holcomb et al. 1988; Chang and Slayman 1991). Already in the ER, Pmal forms a large 1.8MDa homo-oligomeric complex that resists extraction by detergents (Lee et al. 2002). This protein-lipid complex is then packaged into coat protein complex II transport vesicles (Roberg et al. 1999). From the Golgi complex, Pmal is transported to the cell surface by a branch of the secretory pathway that does not intersect with endosomes (Gurunathan et al. 2002; Harsay and Schekman 2002). At the cell surface, Pmal becomes stabilized by a poorly characterized mechanism and occupies detergent-resistant domains that are distinct from those occupied by the arginine/ $\mathrm{H}^{+}$symporter Can $1 \mathrm{p}$ (Bagnat et al. 2000; Malinska et al. 2003). From the plasma membrane, Pmal is finally recycled and turned-over by endocytic delivery to the vacuole.

\section{Stable surface transport of the $\mathrm{H}^{+}$-ATPase depends on ongoing sphingolipid synthesis}

Similar to the synthesis of integral membrane proteins, the synthesis of sphingolipids begins in the ER, where serine palmitoyltransferase catalyzes the condensation of serine with palmitoyl-CoA to form a long-chain base. This longchain base then condenses with a $\mathrm{C} 26$ very-long-chain fatty acid to form ceramide; a reaction that is catalyzed by the ER-localized ceramide synthase. Ceramide is transported from the ER to the Golgi apparatus by vesicular and nonvesicular routes before it is converted to more complex sphingolipids (Levine et al. 2000; Funato and Riezman 2001). Mature sphingolipids are then transported to the plasma membrane, where they are highly enriched (Schneiter 1999; Dickson et al. 2006).

Work by the Schekman and Chang laboratories has established that the biogenesis and stable surface transport of Pmal depends on ongoing sphingolipid synthesis. Using either a temperature-sensitive allele of serine palmitoyltransferase, $l c b 1-100$, or myriocin to block serine palmitoyltransferase activity, these groups showed that 
ongoing long-chain base synthesis is required for oligomerization of Pmal in the ER membrane and for its association with lipid rafts (Lee et al. 2002). In the absence of long-chain base synthesis, monomeric, nonraft-associated Pmal is still exported from the ER, but it is mistargeted to the vacuole where it is degraded (Bagnat et al. 2001; Lee et al. 2002).

We observed that Pmal was rapidly degraded in cells that fail to elongate the ceramide-bound C22 fatty acid to the mature $\mathrm{C} 26$ very-long-chain fatty acid, as is the case in cells lacking ELO3, a component of the ER-localized acyl chain elongase (Oh et al. 1997; Eisenkolb et al. 2002). Interestingly, this rapid turnover of Pmal in the elo3 $\Delta$ mutant is correlated with a lack of the newly synthesized protein to acquire detergent resistance (Eisenkolb et al. 2002). Turnover of Pmal in elo3 $\Delta$ is dependent on ongoing endocytosis, indicating that the protein reaches the plasma membrane first, but that it fails to become stabilized there and instead is endocytosed and delivered to the vacuole for degradation (Eisenkolb et al. 2002) (Fig. 2).

More precise analysis of the requirement for sphingolipids in surface transport and stabilization of Pma1 subsequently revealed that all mutations that affect C26 synthesis result in the rapid turnover of newly synthesized Pmal (Gaigg et al. 2005). Increased turnover of Pmal in these mutants is always accompanied by a failure of the newly synthesized protein to acquire detergent resistance (Gaigg et al. 2005). Remarkably, other mutations that affect the structure of the sphingolipid headgroup or its hydroxylation pattern did not affect raft association or turnover of Pmal (Gaigg et al. 2005). These results thus suggested that the synthesis of C26-containing lipids, rather than ceramide or sphingolipids per se, is important for raft association of newly synthesized Pmal and for its stable delivery to the cell surface. To test this hypothesis, we took advantage of a strain that is viable without synthesizing long-chain base or ceramide and sphingolipids (Dickson et al. 1990). This socalled suppressor strain bears a dominant mutation in an acyltransferase, Slc1, that allows the enzyme to synthesize unusual lipids containing a C26 fatty acid attached to a glycerophospholipid, phosphatidylinositol (PI). These C26containing PIs thus replace the essential function of sphingolipids and structurally and functionally mimic sphingolipids (Lester et al. 1993). Remarkably, analysis of Pmal stability in this suppressor strain revealed that newly synthesized ATPase acquires detergent resistance and that it is stably delivered to the cell surface (Gaigg et al. 2006). Shortening the C26 fatty acid on these suppressor lipids by means of an elo3 $\Delta$ mutation, however, neutralized the suppressor activity of these lipids and resulted in the rapid turnover of the newly synthesized Pmal (Gaigg et al. 2006). These results thus strongly indicate that lipids containing C26 fatty acids, either bound to ceramide or glycerophospholipids, are important for the stable biogenesis of Pmal (Toulmay and Schneiter 2006).

\section{Possible functions of C26-containing lipids}

C26-containing sphingolipids are the hallmark of the yeast plasma membrane. Synthesis of these lipids, however, begins in the ER where both the fatty acid elongase and the ceramide synthase are located. Thus, like the integral membrane proteins that are destined to the cell surface, C26-containing ceramide/sphingolipids must travel from the ER to the plasma membrane. The fact that these lipids affect detergent solubility of newly synthesized Pmal already upon ER exit would indicate that lipids and protein already assemble at their site of synthesis and are then cotransported to the surface (Lee et al. 2002). A failure to properly assemble this protein lipid complex results in a diversion of surface-destined vesicle to the vacuole, a failure in stabilization of the complex upon arrival at the plasma membrane, or both (Bagnat et al. 2001; Gong and Chang 2001; Wang and Chang 2002).

Our observations would indicate that C26-containing lipids are essential for the formation of functional lipidprotein complexes. The precise role that C26 fulfills in this assembly, however, remains to be defined. It has been suggested that the length of the transmembrane domain of proteins along the secretory pathway may increase to match bilayers of increasing "thickness" (Levine et al. 2000). In such a model, the abundance of C26-containing lipids may determine the thickness of membranes along the secretory pathway and thereby affect the sorting of integral membrane proteins. Biophysical studies with pure lipid bilayers, on the other hand, indicate that lipids with highly asymmetric acyl chains can interdigitate into the hydrophobic core of the opposite half of the bilayer and thus do not necessarily increase the thickness of the bilayer (Hui et al. 1984). In addition, membrane proteins themselves have recently been shown to modulate bilayer thickness by more than 10\% (Mitra et al. 2004), an observation that is consistent with the proposition that the thickness of the lipid component of a biological membrane must not naturally match that of the embedded proteins (Mouritsen and Bloom 1993). Based on these considerations, we believe it is premature to correlate acyl chain length with bilayer thickness in biological membranes. Alternative functions of the C26 acyl chains could be to interdigitate into the opposite leaflet, thereby (1) coupling the two halves of the bilayer to lower the energy required to deform this potentially stiff, cholesterol-rich membrane and (2) increase acyl chain packing density to prevent permeability by small molecules. One of the 


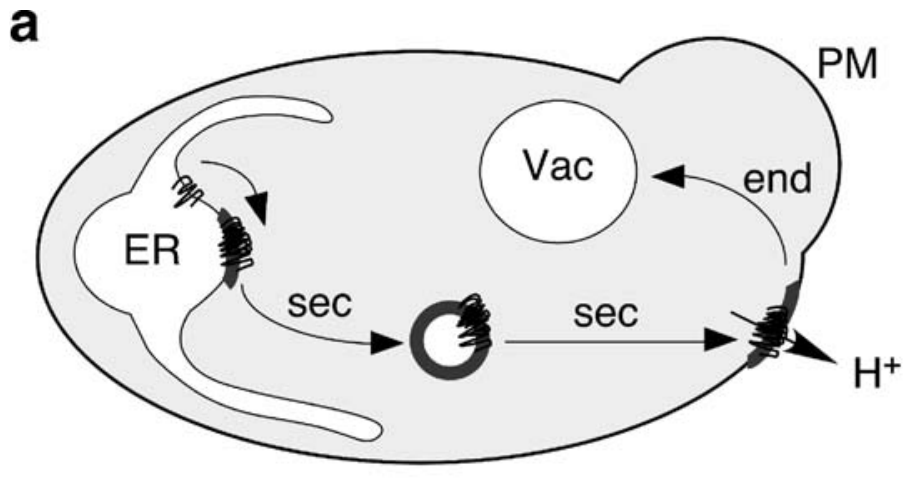

b

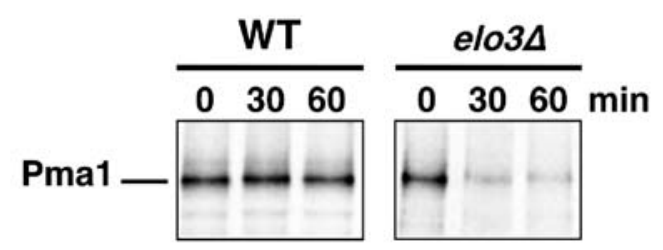

C
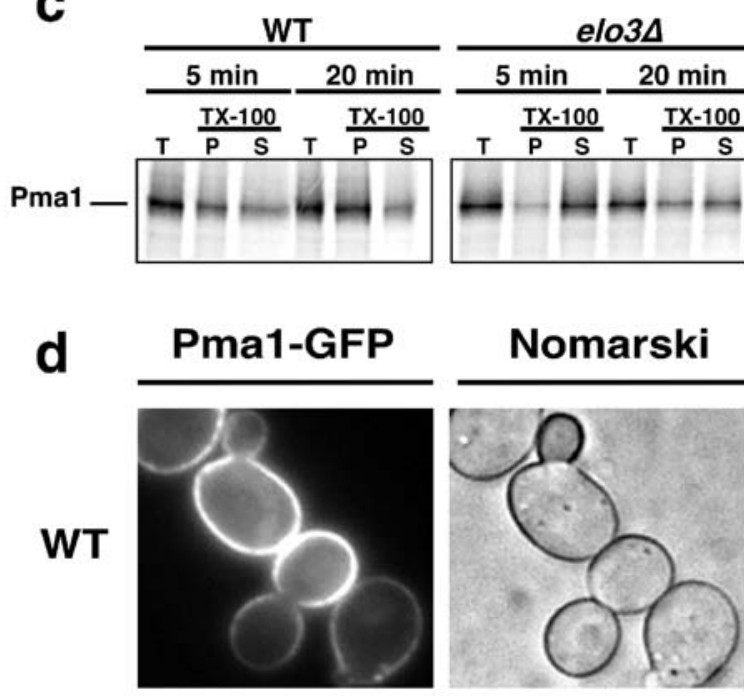

Nomarski

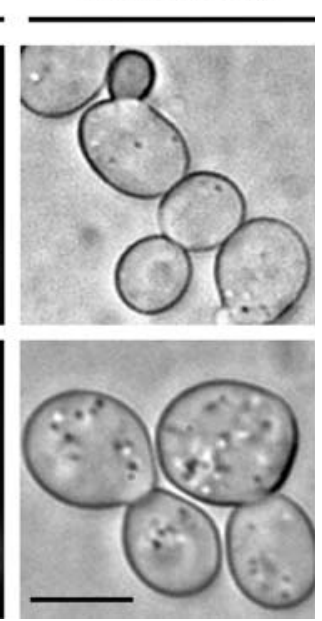

become raft-associated in elongase mutant cells. Pulse-chase analysis followed by detergent extraction reveals that Pmal remains Tritonextractable in elo $3 \Delta$ mutant cells. $T$ total extract, $P$ detergent-resistant pellet fraction, $S$ detergent soluble fraction. The time indicates time after synthesis of Pmal. d Pmal is degraded by routing to the vacuole in elongase mutants. Green fluorescent protein-tagged Pma1 localizes to the plasma membrane in wild-type cells, but is targeted to the vacuolar lumen in elo3 $\Delta$ mutants (arrowheads) (adapted from Toulmay and Schneiter 2006). Bar, $5 \mu \mathrm{m}$

membrane sterol. Mutant cells that are deficient in a late step along the ergosterol biosynthetic pathway due to a defect in the $S$-adenosylmethionine $\Delta 24$ methyltransferase, Erg6, were known to have a reduced capacity to take up tryptophan (Gaber et al. 1989). In an erg6 mutant, however, Tat2 is missorted to the vacuole even if tryptophan levels are low, thus explaining their reduced capacity to take up tryptophan (Umebayashi and Nakano 2003). Remarkably, sorting of Tat 2 to the plasma membrane depends on its association with detergent-insoluble membrane domains, suggesting that sterols affect sorting of Tat 2 through their organization of lipid rafts. In addition, erg6 mutants promote the sorting of Tat 2 into the multivesicular body pathway in late endosomes, which results in the exclusive delivery of Tat 2 into the lumen of the vacuole rather than the limiting membrane of the vacuole, as is observed in wild-type cells (Umebayashi and Nakano 2003). Sterol composition is thus crucial for protein sorting late in the secretory pathway. Sterol-dependent missorting of Tat2 is mediated by polyubiquitination, which is known to act as a 
vacuolar targeting signal, and the inhibition of polyubiquitination restores sorting of Tat 2 to the plasma membrane in an erg6 mutant (Umebayashi and Nakano 2003). Missorting of integral membrane proteins in sterol mutants is not limited to Tat2 but has also been observed for Fus1, a plasma membrane protein required for yeast mating, and an artificial fusion protein, suggesting that membrane sterols play an important role in protein sorting along the exocytic pathway (Bagnat and Simons 2002; Proszynski et al. 2005).

\section{General considerations on the role of lipids in modulating the biogenesis of integral membrane proteins}

The above cases illustrate how lipids can affect the fate of an integral membrane protein in vivo. Given that the membrane lipid composition varies greatly between different organisms, nonfunctional lipid-protein interactions may explain some of the difficulties encountered in expressing functional membrane proteins in heterologous organisms. For example, expression of the mammalian P-glycoprotein in $E$. coli results in nonfunctional protein with a topological inversion of the $\mathrm{C}$-terminal half, including the nucleotidebinding domain (Linton and Higgins 2002). Similarly, a citrate carrier of Klebsiella pneumoniae displays 11 transmembrane domains when inserted into dog pancreas ER membranes but only nine transmembrane domains when expressed in E. coli (van Geest et al. 1999). Thus, the lipid composition of the membranes of the host may be one additional parameter to consider when choosing an organism for the heterologous expression of integral membrane proteins (Opekarova and Tanner 2003). Alternatively, modulation of the host's lipid repertoire either by genetic means or by supplementation of soluble lipid precursors may be instrumental to recover a functional membrane protein (Dowhan 1997).

Acknowledgements We thank Rashi Tiwari for careful proofreading of this manuscript and the Swiss National Science Foundation (PP00A-110450) for financial support. We apologize for the primary literature that could not be cited due to space limitations.

\section{References}

Abramson J, Smirnova I, Kasho V, Verner G, Kaback HR, Iwata S (2003) Structure and mechanism of the lactose permease of Escherichia coli. Science 301:610-615

Bagnat M, Simons K (2002) Cell surface polarization during yeast mating. Proc Natl Acad Sci USA 99:14183-14188

Bagnat M, Keranen S, Shevchenko A, Simons K (2000) Lipid rafts function in biosynthetic delivery of proteins to the cell surface in yeast. Proc Natl Acad Sci USA 97:3254-3259
Bagnat M, Chang A, Simons K (2001) Plasma membrane proton ATPase Pma1p requires raft association for surface delivery in yeast. Mol Biol Cell 12:4129-4138

Birner R, Burgermeister M, Schneiter R, Daum G (2001) Roles of phosphatidylethanolamine and of its several biosynthetic pathways in Saccharomyces cerevisiae. Mol Biol Cell 12:9971007

Bogdanov M, Dowhan W (1995) Phosphatidylethanolamine is required for in vivo function of the membrane-associated lactose permease of Escherichia coli. J Biol Chem 270:732-739

Bogdanov M, Umeda M, Dowhan W (1999) Phospholipid-assisted refolding of an integral membrane protein. Minimum structural features for phosphatidylethanolamine to act as a molecular chaperone. J Biol Chem 274:12339-12345

Bogdanov M, Heacock PN, Dowhan W (2002) A polytopic membrane protein displays a reversible topology dependent on membrane lipid composition. EMBO J 21:2107-2116

Bowie JU (2005) Solving the membrane protein folding problem. Nature 438:581-589

Chang A, Slayman CW (1991) Maturation of the yeast plasma membrane $[\mathrm{H}+]$ ATPase involves phosphorylation during intracellular transport. J Cell Biol 115:289-295

Chen CC, Wilson TH (1984) The phospholipid requirement for activity of the lactose carrier of Escherichia coli. J Biol Chem 259:10150-10158

Dickson RC, Wells GB, Schmidt A, Lester RL (1990) Isolation of mutant Saccharomyces cerevisiae strains that survive without sphingolipids. Mol Cell Biol 10:2176-2181

Dickson RC, Sumanasekera C, Lester RL (2006) Functions and metabolism of sphingolipids in Saccharomyces cerevisiae. Prog Lipid Res 45(6):447-465

Dowhan W (1997) Molecular basis for membrane phospholipid diversity: why are there so many lipids? Annu Rev Biochem 66:199-232

Dowhan W, Mileykovskaya E, Bogdanov M (2004) Diversity and versatility of lipid-protein interactions revealed by molecular genetic approaches. Biochim Biophys Acta 1666:19-39

Dunlop J, Jones PC, Finbow ME (1995) Membrane insertion and assembly of ductin: a polytopic channel with dual orientations. EMBO J 14:3609-3616

Eisenkolb M, Zenzmaier C, Leitner E, Schneiter R (2002) A specific structural requirement for ergosterol in long-chain fatty acid synthesis mutants important for maintaining raft domains in yeast. Mol Biol Cell 13:4414-4428

Epand RM (1998) Lipid polymorphism and protein-lipid interactions. Biochim Biophys Acta 1376:353-368

Funato K, Riezman H (2001) Vesicular and nonvesicular transport of ceramide from ER to the Golgi apparatus in yeast. J Cell Biol 155:949-959

Gaber RF, Copple DM, Kennedy BK, Vidal M, Bard M (1989) The yeast gene ERG6 is required for normal membrane function but is not essential for biosynthesis of the cell-cycle-sparking sterol. Mol Cell Biol 9:3447-3456

Gaigg B, Timischl B, Corbino L, Schneiter R (2005) Synthesis of sphingolipids with very long chain fatty acids but not ergosterol is required for routing of newly synthesized plasma membrane ATPase to the cell surface of yeast. J Biol Chem 280:2251522522

Gaigg B, Toulmay A, Schneiter R (2006) Very long-chain fatty acidcontaining lipids rather than sphingolipids per se are required for raft association and stable surface transport of newly synthesized plasma membrane ATPase in yeast. J Biol Chem (in press) DOI 10.1074/jbc.M603791200

Gong X, Chang A (2001) A mutant plasma membrane ATPase, Pma110 , is defective in stability at the yeast cell surface. Proc Natl Acad Sci USA 98:9104-9109 
Gurunathan S, David D, Gerst JE (2002) Dynamin and clathrin are required for the biogenesis of a distinct class of secretory vesicles in yeast. EMBO J 21:602-614

Harsay E, Schekman R (2002) A subset of yeast vacuolar protein sorting mutants is blocked in one branch of the exocytic pathway. J Cell Biol 156:271-285

Hegde RS, Lingappa VR (1999) Regulation of protein biogenesis at the endoplasmic reticulum membrane. Trends Cell Biol 9:132-137

Hessa T, Kim H, Bihlmaier K, Lundin C, Boekel J, Andersson H, Nilsson I, White SH, von Heijne G (2005) Recognition of transmembrane helices by the endoplasmic reticulum translocon. Nature 433:377-381

Holcomb CL, Hansen WJ, Etcheverry T, Schekman R (1988) Secretory vesicles externalize the major plasma membrane ATPase in yeast. J Cell Biol 106:641-648

Hui SW, Mason TJ, Huang C-H (1984) Acyl chain interdigitation in saturated mixed chain phosphatidylcholine bilayer dispersions. Biochemistry 23:5570-5577

Jensen MO, Mouritsen OG (2004) Lipids do influence protein function - the hydrophobic matching hypothesis revisited. Biochim Biophys Acta 1666:205-226

Krogh A, Larsson B, von Heijne G, Sonnhammer EL (2001) Predicting transmembrane protein topology with a hidden Markov model: application to complete genomes. J Mol Biol 305:567-580

Kühlbrand W (2004) Biology, structure and mechanism of P-type ATPases. Nat Rev Mol Cell Biol 5:282-295

Lambert C, Prange R (2001) Dual topology of the hepatitis B virus large envelope protein: determinants influencing post-translational pre-S translocation. J Biol Chem 276:22265-22272

Lee AG (2004) How lipids affect the activities of integral membrane proteins. Biochim Biophys Acta 1666:62-87

Lee MC, Hamamoto S, Schekman R (2002) Ceramide biosynthesis is required for the formation of the oligomeric $\mathrm{H}+$-ATPase Pmalp in the yeast endoplasmic reticulum. J Biol Chem 277:22395-22401

Lester RL, Wells GB, Oxford G, Dickson RC (1993) Mutant strains of Saccharomyces cerevisiae lacking sphingolipids synthesize novel inositol glycerophospholipids that mimic sphingolipid structures. J Biol Chem 268:845-856

Levine TP, Wiggins CA, Munro S (2000) Inositol phosphorylceramide synthase is located in the Golgi apparatus of Saccharomyces cerevisiae. Mol Biol Cell 11:2267-2281

Levy D (1996) Membrane proteins which exhibit multiple topological orientations. Essays Biochem 31:49-60

Linton KJ, Higgins CF (2002) P-glycoprotein misfolds in Escherichia coli: evidence against alternating-topology models of the transport cycle. Mol Membr Biol 19:51-58

Lu Y, Turnbull IR, Bragin A, Carveth K, Verkman AS, Skach WR (2000) Reorientation of aquaporin-1 topology during maturation in the endoplasmic reticulum. Mol Biol Cell 11:2973-2985

Malinska K, Malinsky J, Opekarova M, Tanner W (2003) Visualization of protein compartmentation within the plasma membrane of living yeast cells. Mol Biol Cell 14:4427-4436

McMahon HT, Gallop JL (2005) Membrane curvature and mechanisms of dynamic cell membrane remodelling. Nature 438:590-596

Mitra K, Ubarretxena-Belandia I, Taguchi T, Warren G, Engelman DM (2004) Modulation of the bilayer thickness of exocytic pathway membranes by membrane proteins rather than cholesterol. Proc Natl Acad Sci USA 101:4083-4088

Mouritsen OG, Bloom M (1993) Models of lipid-protein interactions in membranes. Annu Rev Biophys Biomol Struct 22:145-171

Oh CS, Toke DA, Mandala S, Martin CE (1997) ELO2 and ELO3, homologues of the Saccharomyces cerevisiae ELO1 gene, function in fatty acid elongation and are required for sphingolipid formation. J Biol Chem 272:17376-17384
Opekarova M, Tanner W (2003) Specific lipid requirements of membrane proteins - a putative bottleneck in heterologous expression. Biochim Biophys Acta 1610:11-22

Opekarova M, Robl I, Tanner W (2002) Phosphatidyl ethanolamine is essential for targeting the arginine transporter Can $1 \mathrm{p}$ to the plasma membrane of yeast. Biochim Biophys Acta 1564:9-13

Opekarova M, Malinska K, Novakova L, Tanner W (2005) Differential effect of phosphatidylethanolamine depletion on raft proteins: further evidence for diversity of rafts in Saccharomyces cerevisiae. Biochim Biophys Acta 1711:87-95

Osborne AR, Rapoport TA, van den Berg B (2005) Protein translocation by the Sec61/SecY channel. Annu Rev Cell Dev Biol 21:529-550

Palsdottir H, Hunte C (2004) Lipids in membrane protein structures. Biochim Biophys Acta 1666:2-18

Pornillos O, Chang G (2006) Inverted repeat domains in membrane proteins. FEBS Lett 580:358-362

Proszynski TJ, Klemm RW, Gravert M, Hsu PP, Gloor Y, Wagner J, Kozak K, Grabner H, Walzer K, Bagnat M, Simons K, WalchSolimena C (2005) A genome-wide visual screen reveals a role for sphingolipids and ergosterol in cell surface delivery in yeast. Proc Natl Acad Sci USA 102:17981-17986

Rapp M, Granseth E, Seppala S, von Heijne G (2006) Identification and evolution of dual-topology membrane proteins. Nat Struct Mol Biol 13:112-116

Roberg KJ, Crotwell M, Espenshade P, Gimeno R, Kaiser CA (1999) LST1 is a SEC24 homologue used for selective export of the plasma membrane ATPase from the endoplasmic reticulum. J Cell Biol 145:659-672

Robl I, Grassl R, Tanner W, Opekarova M (2001) Construction of phosphatidylethanolamine-less strain of Saccharomyces cerevisiae. Effect on amino acid transport. Yeast 18:251-260

Schleiff E, Tien R, Salomon M, Soll J (2001) Lipid composition of outer leaflet of chloroplast outer envelope determines topology of OEP7. Mol Biol Cell 12:4090-4102

Schneiter R (1999) Brave little yeast, please guide us to Thebes: sphingolipid function in S. cerevisiae. Bioessays 21:1004-1010

Seto-Young D, Chen CC, Wilson TH (1985) Effect of different phospholipids on the reconstitution of two functions of the lactose carrier of Escherichia coli. J Membr Biol 84:259-267

Storey MK, Clay KL, Kutateladze T, Murphy RC, Overduin M, Voelker DR (2001) Phosphatidylethanolamine has an essential role in Saccharomyces cerevisiae that is independent of its ability to form hexagonal phase structures. J Biol Chem 276:48539-48548

Toulmay A, Schneiter R (2006) Lipid-dependent surface transport of the proton pumping ATPase: a model to study plasma membrane biogenesis in yeast. Biochimie (in press)

Umebayashi K, Nakano A (2003) Ergosterol is required for targeting of tryptophan permease to the yeast plasma membrane. J Cell Biol 161:1117-1131

van Dalen A, de Kruijff B (2004) The role of lipids in membrane insertion and translocation of bacterial proteins. Biochim Biophys Acta 1694:97-109

Van den Berg B, Clemons WM Jr, Collinson I, Modis Y, Hartmann E, Harrison SC, Rapoport TA (2004) X-ray structure of a proteinconducting channel. Nature 427:36-44

van Geest M, Nilsson I, von Heijne G, Lolkema JS (1999) Insertion of a bacterial secondary transport protein in the endoplasmic reticulum membrane. J Biol Chem 274:2816-2823

van Klompenburg W, Nilsson I, von Heijne G, de Kruijff B (1997) Anionic phospholipids are determinants of membrane protein topology. EMBO J 16:4261-4266

Wang Q, Chang A (2002) Sphingoid base synthesis is required for oligomerization and cell surface stability of the yeast plasma membrane ATPase, Pma1. Proc Natl Acad Sci USA 99:12853-12858 
Wang X, Bogdanov M, Dowhan W (2002) Topology of polytopic membrane protein subdomains is dictated by membrane phospholipid composition. EMBO J 21:5673-5681

Wright R, Basson M, D'Ari L, Rine J (1988) Increased amounts of HMG-CoA reductase induce "karmellae": a proliferation of stacked membrane pairs surrounding the yeast nucleus. J Cell Biol 107:101-114

Xie J, Bogdanov M, Heacock P, Dowhan W (2006) Phosphatidylethanolamine and monoglucosyldiacylglycerol are interchangeable in supporting topogenesis and function of the polytopic membrane protein lactose permease. J Biol Chem 281:1917219178
Zhang JT, Duthie M, Ling V (1993) Membrane topology of the Nterminal half of the hamster P-glycoprotein molecule. J Biol Chem 268:15101-15110

Zhang W, Bogdanov M, Pi J, Pittard AJ, Dowhan W (2003) Reversible topological organization within a polytopic membrane protein is governed by a change in membrane phospholipid composition. J Biol Chem 278:50128-50135

Zhang W, Campbell HA, King SC, Dowhan W (2005) Phospholipids as determinants of membrane protein topology. Phosphatidylethanolamine is required for the proper topological organization of the gamma-aminobutyric acid permease (GabP) of Escherichia coli. J Biol Chem 280:26032-26038 